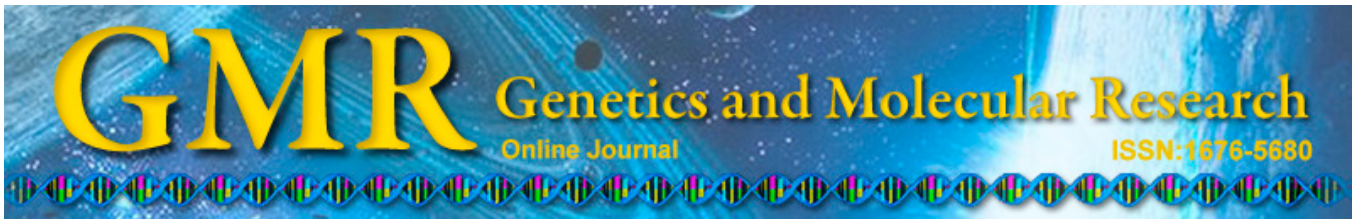

\title{
Inhibition of human chronic myelogenous leukemia K562 cell growth following combination treatment with resveratrol and imatinib mesylate
}

\author{
X.J. Wang ${ }^{1,2}$ and Y.H. Li ${ }^{1}$ \\ ${ }^{1}$ Department of Hematology, ZhuJiang Hospital of Southern Medical University, \\ Guangdong, Guangzhou, China \\ ${ }^{2}$ Department of Hematology, Ankang City Central Hospital, Shaanxi, \\ Ankang, China \\ Corresponding author: Y.H. Li \\ E-mail: liyuhua108@126.com
}

Genet. Mol. Res. 14 (2): 6413-6418 (2015)

Received October 14, 2014

Accepted February 10, 2015

Published June 11, 2015

DOI http://dx.doi.org/10.4238/2015.June.11.17

\begin{abstract}
To investigate the effect of treatment with resveratrol combined with imatinib mesylate on human chronic myelogenous leukemia K562 cell growth inhibition and apoptosis, in vitro cultured human chronic myelogenous leukemia K562 cells were incubated with different concentrations of resveratrol and imatinib mesylate when the cells were in the logarithmic phase. Next, the cell growth inhibition was evaluated using the MTT assay and cellular morphology observation. Apoptosis was determined using Annexin V fluorescein isothiocyanate/ propidium iodide double staining. The results demonstrated that treatment with resveratrol (concentration-dependent) and imatinib mesylate showed significantly greater inhibition of K562 cell growth and a higher apoptosis rate of K562 cells than imatinib mesylate medication alone and the control group $(\mathrm{P}<0.01)$. The imatinib mesylate medication alone group showed significant inhibition of K562
\end{abstract}


cell growth and apoptosis rate of K562 cells compared to the control group $(\mathrm{P}<0.01)$. Our findings indicate that imatinib mesylate and resveratrol are potent drug treatments for human chronic myelogenous leukemia, offering a promising means of inhibiting cell growth and apoptosis.

Key words: Chronic myeloid leukemia; Growth and proliferation; K562 cell; Imatinib mesylate; Resveratrol

\section{INTRODUCTION}

Chronic myelogenous leukemia (CML) is a malignant, clonal hematopoietic stem cell disorder, accounting for $20 \%$ of all leukemia cases, with an annual incidence of 1-1.5 cases per 100,000 and a wide age distribution. The incidence of this disease gradually increases with age (Tong et al., 2012).

CML is characterized by the presence of a breakpoint cluster region (BCR)-Abelson murine leukemia $(\mathrm{ABL})$ fusion gene, which results from reciprocal translocation between chromosomes 9 and 22, cytogenetically visible as a shortened chromosome 22 (Philadelphia chromosome) (Kantarjian et al., 2007).

The essential role of BCR-ABL tyrosine kinase activity in cellular transformation provides the rational for targeting this function therapeutically (Druker et al., 2001). Treatment with imatinib is accompanied with many toxic side effects as well as drug resistance. Thus, a new drug treatment against CML is needed (Kantarjian et al., 2007). A previous study showed that resveratrol could protect against cancer (Bishayee et al., 2010). Therefore, the purpose of the present study was to investigate the anti-CML effects of imatinib and resveratrol on human K562 cell proliferation and apoptosis.

\section{MATERIAL AND METHODS}

\section{Reagents}

K562 was purchased from Shanghai Bogoo Biotechnology. Co., Ltd. (Shanghai, China) and dissolved in dimethyl sulfoxide. Resveratrol was purchased from Hubei Jusheng Technology Co., Ltd. (Hubei, China). Imatinib was purchased from Novartis (Basel, Switzerland).

Propidium iodide (PI) and 3-(4,5-dimethylthiazol-2yl)-2,5-diphenyl tetrazolium bromide (MTT) were purchased from Sigma-Aldrich (St. Louis, MO, USA). The annexin Vfluorescein isothiocyanate (FITC)/PI apoptosis detection kit was purchased from KeyGEN Biotech (NanJing, China).

\section{Cell culture}

The K562 cell line was purchased from the Cell Bank of the Chinese Academy of Sciences (Shanghai, China) and cultured in RPMI 1640 medium supplemented with 10\% fetal bovine serum (GIBCO, Grand Island, NY, USA) at $37^{\circ} \mathrm{C}$ containing $5 \% \mathrm{CO}_{2}$. 


\section{MTT assay}

Cells were seeded on 96-well plates overnight and then incubated with various concentrations of resveratrol $(1.5,3.1,6.2,12.5,18.7,25.0,37.5$, and $50.0 \mu \mathrm{M})$ with or without $0.5 \mu \mathrm{M}$ imatinib for $48 \mathrm{~h}$. Each drug concentration was administered in 6 replicate wells. A negative control group without drugs was also established. At the end of the treatment, $20 \mu \mathrm{L}$ $5 \mathrm{mg} / \mathrm{mL}$ MTT solution was added to each well and incubated at $37^{\circ} \mathrm{C}$ for $4 \mathrm{~h}$. Next, $150 \mu \mathrm{L}$ dimethyl sulfoxide was added to each well after the medium was removed and mixed by agitation at room temperature for $10 \mathrm{~min}$. The optical density values were measured at $490 \mathrm{~nm}$ on a scanning multiwall spectrophotometer (Model 550, Bio-Rad, Hercules, CA, USA).

\section{Flow cytometry detection of apoptotic cells}

The cells, both adherent and suspended, were collected and washed twice with phosphate-buffered saline. The annexin V-FITC/PI apoptosis detection kit was used according to the manufacturer protocol. After annexin V-FITC was added into the labeled tube for $5 \mathrm{~min}$, PI was added and incubated for $15 \mathrm{~min}$ in the dark. Samples were analyzed using FACScan flow cytometry and the CellQuest analysis software (BD Biosciences, Franklin Lakes, NJ, USA). Data analysis was performed using the Cellquest software 1.2 version.

\section{Statistical analysis}

Statistical analysis was performed using the SPSS 20.0 software (SPSS, Inc., Chicago, IL, USA). The results are reported as means \pm standard deviation (SD), and $\mathrm{P}$ values $<0.05$ were considered to be statistically significant. Each experiment consisted of at least 3 replicates per condition.

\section{RESULTS}

\section{Combination drugs inhibit the colony formation ability of K562 cells}

After $48 \mathrm{~h}$ of drug treatment, we determined the value of colony formation. As shown in Figure 1, the colony formation of K562 cell line was dramatically suppressed upon both resveratrol and imatinib treatment.

A

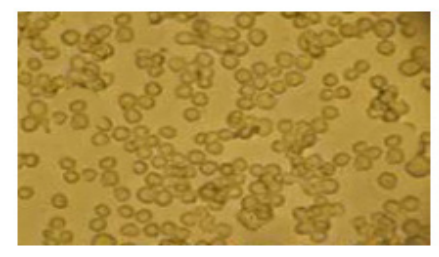

B

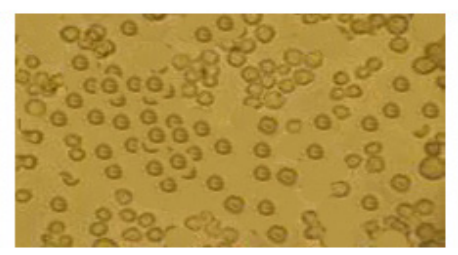

C

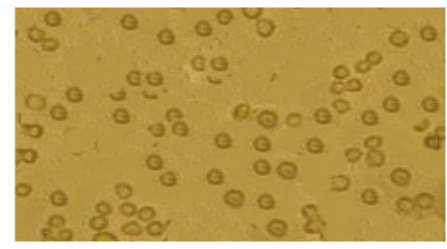

Figure 1. Drug treatment inhibits the colony formation ability of K562 cells. After treatment with resveratrol with or without imatinib for $48 \mathrm{~h}$, the cells were washed twice with phosphate-buffered saline, fixed with $4 \%$ paraformaldehyde for $10 \mathrm{~min}$, stained with crystal violet for $10 \mathrm{~min}$, washed again, and photographed. A. Control group. B. Treatment with imatinib. C. Treatment with resveratrol with or without imatinib. 


\section{Treatment with resveratrol and imatinib inhibit $\mathrm{K562}$ cell proliferation}

As shown in Table 1, the cell viability in the $50 \mu \mathrm{M}$ resveratrol treatment and $0.5 \mu \mathrm{M}$ imatinib treatment group showed the most significant decrease in contrast to that observed in the $0.5 \mu \mathrm{M}$ imatinib treatment group and the control group $(\mathrm{P}<0.5)$. Inhibition of cell proliferation in the combined treatment group showed concentration-dependence on resveratrol.

Table 1. Determination of the inhibition of cell growth through drug treatment using MTT method in each group.

\begin{tabular}{lccc}
\hline Group & N (case) & SI & \% Reduction* \\
\hline Control & 3 & $0.87 \pm 0.03$ & 0 \\
Imatinib treatment & 3 & $0.78 \pm 0.01$ & $10.01 \pm 4.06$ \\
Imatinib + resveratrol 1 & 3 & $0.70 \pm 0.02$ & $19.31 \pm 5.12$ \\
Imatinib + resveratrol 2 & 3 & $0.66 \pm 0.03$ & $24.21 \pm 6.14$ \\
Imatinib + resveratrol 3 & 3 & $0.59 \pm 0.01$ & $32.03 \pm 4.06$ \\
Imatinib + resveratrol 4 & 3 & $0.50 \pm 0.02$ & $44.04 \pm 4.07$ \\
Imatinib + resveratrol 5 & 3 & $0.45 \pm 0.03$ & $48.02 \pm 5.22$ \\
Imatinib + resveratrol 6 & 3 & $0.39 \pm 0.01$ & $55.02 \pm 3.26$ \\
Imatinib + resveratrol 7 & 3 & $0.30 \pm 0.02$ & $65.01 \pm 4.01$ \\
Imatinib + resveratrol 8 & 3 & $0.27 \pm 0.02$ & $69.01 \pm 4.02$ \\
\hline
\end{tabular}

$\mathrm{SI}=$ stimulation index. $*$ Reduction of SI compared to blank control group values.

\section{K562 cell apoptosis}

To investigate whether the growth inhibitory effect was mediated through the induction of apoptosis, annexin V-FITC/PI double-staining assay was used to assess the apoptosisinducing effect of MK-2206. As shown in Table 2, K562 cells treated with $50 \mu \mathrm{M}$ resveratrol and $0.5 \mu \mathrm{M}$ imatinib displayed a higher apoptosis rate than that observed in the $0.5 \mu \mathrm{M}$ imatinib treatment group and the control group $(\mathrm{P}<0.5)$. The apoptosis rate in the combination treatment group showed a concentration.

Table 2. Apoptosis rate of each group analyzed using flow cytometry.

\begin{tabular}{lcr}
\hline Group & $\mathrm{N}$ (case) & Apoptosis rate (\%) \\
\hline Control & 3 & $3.53 \pm 0.02$ \\
Imatinib treatment & 3 & $10.01 \pm 0.01$ \\
Imatinib + resveratrol 1 & 3 & $11.99 \pm 0.02$ \\
Imatinib + resveratrol 2 & 3 & $12.08 \pm 0.10$ \\
Imatinib + resveratrol 3 & 3 & $13.01 \pm 0.01$ \\
Imatinib + resveratrol 4 & 3 & $14.02 \pm 0.20$ \\
Imatinib + resveratrol 5 & 3 & $14.99 \pm 0.26$ \\
Imatinib + resveratrol 6 & 3 & $15.01 \pm 0.07$ \\
Imatinib + resveratrol 7 & 3 & $15.88 \pm 0.12$ \\
Imatinib + resveratrol 8 & 3 & $16.55 \pm 0.23$ \\
\hline
\end{tabular}

\section{DISCUSSION}

Most CML cases are associated with the presence of a specific chromosomal translocation $(9 ; 22)$ (q34; q11) (O’Dwyer and Druker, 2001). This reciprocal translocation between the long arms of chromosomes 9 and 22 results in a shortened chromosome 22, commonly known as the Philadelphia chromosome. The molecular consequence of this translocation 
event is the fusion of the c- $a b l$ oncogene from chromosome 9 to sequences from chromosome 22 , the $b c r$, giving rise to a chimeric $b c r-a b l$ gene (Daley et al., 1990).

Numerous factors affect the chronic phase of CML and survival, such as age, white blood cell count, basophilic granulocyte count, liver and spleen size, anemia, and platelet count, among others (Deininger et al., 2000).

Standard treatment options for patients in the chronic phase of CML are allogeneic stem cell transplantation, imatinib, or interferon- $\gamma$-based regimens. Initial cytoreductive therapy often uses hydroxyurea (O'Dwyer and Druker, 2001). This agent, a ribonucleotide reductase inhibitor, is effective for maintaining blood counts in most patients. Hydroxyurea is generally well tolerated, but cytogenetic responses (a reduction in the percentage of Philadelphia-positive bone marrow metaphases) are rare, and the onset to blast crisis is not delayed, with transformation occurring within a median of 4-6 years (O’Dwyer et al., 2002).

Allogeneic stem cell transplantation, using myeloablative doses of chemotherapy and/ or radiation followed by infusion of allogeneic stem cells, remains the only proven curative therapy for CML (Brunstein and McGlave, 2001), but other factors predicting poorer outcomes from an allogeneic stem cell transplant include increasing age and poorer-quality matches. A potential problem with the use of interferon- $\gamma$ is that it may compromise the outcome of subsequent transplantations through increased graft rejection or graft-versus-host disease unless it has been discontinued for at least 3 months prior to the procedure, but imatinib can retrieve these shortcomings to some extent (O'Dwyer et al., 2002).

More importantly, imatinib inhibited signaling of the ligand-activated platelet-derived growth factor receptor, as determined by ligand-stimulated autophosphorylation of this receptor, at a $50 \%$ inhibitory concentration $\left(\mathrm{IC}_{50}\right)$ of $0.1-1 \mu \mathrm{M}$. Inhibition of the constitutively active TEL-platelet-derived growth factor receptor fusion protein was observed at an $\mathrm{IC}_{50}$ value of $0.15 \mu \mathrm{M}$. Furthermore, the compound potent effect is to inhibit the autophosphorylation of Kit receptor by binding cognate ligand, stem-cell factor, and to suppress Kit autophosphorylation in a cell line established from a patient with a gastrointestinal stromal tumor with an activating Kit mutation. Additionally, treatment with imatinib $(50 \mathrm{mg} / \mathrm{kg}$ in the morning, $100 \mathrm{mg} /$ $\mathrm{kg}$ in the evening) led to prolonged survival of patients (Deininger et al., 2005). However, the popularity of imatinib has been limited because of its unexpected late side effects, cost, the uncertainty of drug withdrawal time, and drug resistance.

Interestingly, resveratrol has been suggested to be one of the most promising cancer chemopreventive agents and has been shown to inhibit cellular events associated with carcinogenesis (Delmas et al., 2006). Recently, the cancer chemopreventive activity of resveratrol has been shown to trigger apoptosis (Kuhnle et al., 2000).

Resveratrol is a traditional oriental medicine that induces apoptosis in some lines of human tumor cells. It constitutes the major digoxin-like immunoreactive component of Chan $\mathrm{Su}$, obtained from the skin and parotid venom glands of toads. Bufalin is a cardio-active C-24 steroid that exhibits a variety of biological activities, such as cardiotonic, anesthetic, blood pressure stimulatory, respiratory, and antineoplastic effects. Bufalin has been demonstrated to inhibit the growth of tumors, such as endometrial and ovarian cancers. The compound is able to mediate the inhibition of cell growth, cell cycle arrest, apoptosis, and expression of genes related to malignant phenotype in human cancer cells (Takai et al., 2010; Hu et al., 2010).

No previous studies have examined combination treatment with resveratrol and imatinib to treat CML. In this study, we found that treatment with resveratrol and imatinib mesylate showed significantly greater inhibition of K562 cell growth and a higher apoptosis rate than 
that with imatinib mesylate treatment alone, but these effects may depend on concentration, suggesting that synergistic drug treatment is characterized as small dosage, moderate curative effect, low side-effects, and no resistance. Based on our results, these 2 drugs may have additive effect on CML, but the effect and suitable dosage should be evaluated in further studies.

In summary, we demonstrated that treatment with imatinib and resveratrol have important effects on K562 cells by inhibiting cell growth and by inducing cell apoptosis. In addition, combination treatment with MK-2206 and TNF-related apoptosis-inducing ligand may represent a novel strategy for overcoming drug resistance, and combination of imatinib and resveratrol may be an effective strategy for human CML therapy. Moreover, the combination of imatinib and resveratrol represents a paradigm shift in cancer drug development. This may result in a new generation of specific, targeted therapies for the numerous malignant conditions for which no efficient drug therapy is currently available.

\section{REFERENCES}

Bishayee A, Politis T and Darvesh AS (2010). Resveratrol in the chemoprevention and treatment of hepatocellular carcinoma. Cancer Treat Rev. 36: 43-53.

Brunstein CG and McGlave PB (2001). The biology and treatment of chronic myelogenous leukemia. Oncology 15: 23-35.

Daley GQ, Van Etten RA and Baltimore D (1990). Induction of chronic myelogenous leukemia in mice by the P210bcr/ abl gene of the Philadelphia chromosome. Science 247: 824-830.

Deininger M, Buchdunger E and Drunker BJ (2005). The development of imatinib as a therapeutic agentfor chronic myeloid leukemia. Blood 105: 2640-2653.

Deininger MW, Vieira S, Mendiola R, Schultheis B, et al. (2000). BCR-ABL tyrosine kinase activity regulates the expression of multiple genes implicated in the pathogenesis of chronic myeloid leukemia. Cancer Res. 60: 2049-2055.

Delmas D, Lançon A, Colin D, Jannin B, et al. (2006). Resveratrol as a chemopreventive agent: a promising molecule for fighting cancer. Curr. Drug Targets 7: 423-442.

Druker BJ, Talpaz M, Resta DJ, Peng B, et al (2001). Efficacy and safety of a specific inhibitor of the BCR-ABL tyrosine kinase in chronic myeloid leukemia. N. Engl. J. Med. 344:1031-1037.

$\mathrm{Hu}$ H, Li Y, Gu J, Zhu X, et al. (2010). Antisense oligonucleotide against miR-21 inhibits migration and induces apoptosis in leukemic K562 cells. Leuk. Lymphoma 51: 694-701.

Kantarjian HM, Giles F, Quintás-Cardama A and Cortes J (2007). Important therapeutic targets in chronic myelogenous leukemia. Clin. Cancer Res. 13: 1089-1097.

Kuhnle G, Spencer JP, Chowrimootoo G, Schroeter H, et al. (2000). Resveratrol is absorbed in the small intestine as resveratrol glucuronide. Biochem. Biophys. Res. Commun. 272: 212-217.

O'Dwyer ME and Druker BJ (2001). Chronic myelogenous leukaemia - new therapeutic principles. J. Intern. Med. 250: 3-9.

O'Dwyer ME, Mauro MJ and Druker BJ (2002). Recent advancements in the treatment of chronic myelogenous leukemia. Annu. Rev. Med. 53: 369-381.

Takai N, Kira N, Ishii T, Yoshida T, et al. (2010). Bufalin, a traditional oriental medicine, induces apoptosis in human cancer cells. Asian Pac. J. Cancer Prev. 13: 399-402.

Tong Y, Liu YY, You LS and Qian WB (2012). Perifosine induces protective autophagy and upregulation of ATG5 in human chronic myelogenous leukemia cells in vitro. Acta Pharmacol. Sin. 33: 542-550. 